\title{
TCL1A, a Novel Transcription Factor and a Coregulator of Nuclear Factor $\kappa$ B p65: Single Nucleotide Polymorphism and Estrogen Dependence ${ }^{[\mathrm{S}}$
}

\author{
Ming-Fen Ho, Edroaldo Lummertz da Rocha, Cheng Zhang, James N. Ingle, Paul E. Goss, \\ Lois E. Shepherd, Michiaki Kubo, Liewei Wang, $\mathrm{Hu} \mathrm{Li}$, and Richard M. Weinshilboum \\ Division of Clinical Pharmacology, Department of Molecular Pharmacology and Experimental Therapeutics (M.H., E.L.d.R., C.Z., \\ L.W., H.L., R.M.W.), and Division of Medical Oncology, Department of Oncology (J.N.I.), Mayo Clinic, Rochester, Minnesota; \\ Division of Hematology/Oncology, Department of Medicine, Massachusetts General Hospital Cancer Center, Boston, \\ Massachusetts (P.E.G.); Canadian Cancer Trials Group, Kingston, Ontario, Canada (L.E.S.); and RIKEN Center for Integrative \\ Medical Science, Yokohama, Japan (M.K.)
}

Received January 10, 2018; accepted March 19, 2018

\section{ABSTRACT}

T-cell leukemia 1A (TCL1A) single-nucleotide polymorphisms (SNPs) have been associated with aromatase inhibitor-induced musculoskeletal adverse events. We previously demonstrated that TCL1A is inducible by estradiol $\left(E_{2}\right)$ and plays a critical role in the regulation of cytokines, chemokines, and Toll-like receptors in a TCL1A SNP genotype and estrogen-dependent fashion. Furthermore, TCLIA SNP-dependent expression phenotypes can be "reversed" by exposure to selective estrogen receptor modulators such as 4-hydroxytamoxifen (4OH-TAM). The present study was designed to comprehensively characterize the role of TCL1A in transcriptional regulation across the genome by performing RNA sequencing (RNA-seq) and chromatin immunoprecipitation sequencing (ChIP-seq) assays with lymphoblastoid cell lines. RNA-seq identified 357 genes that were regulated in a TCL1A SNP-and $\mathrm{E}_{2}$-dependent fashion with expression patterns that were $4 \mathrm{OH}-\mathrm{TAM}$ reversible. ChIP-seq for the same cells identified 57 TCL1A binding sites that could be regulated by $E_{2}$ in a SNP-dependent fashion. Even more striking, nuclear factor $-\kappa \mathrm{B}(\mathrm{NF}-\kappa \mathrm{B})$ p 65 bound to those same DNA regions. In summary, TCL1A is a novel transcription factor with expression that is regulated in a SNP- and $E_{2}$-dependent fashion-a pattern of expression that can be reversed by $4 \mathrm{OH}-\mathrm{TAM}$. Integrated RNA-seq and ChIP-seq results suggest that TCL1A also acts as a transcriptional coregulator with NF- $\kappa \mathrm{B}$ p65, an important immune system transcription factor.

\section{Introduction}

We previously performed a genome-wide association study (GWAS) that identified three single-nucleotide polymorphisms (SNPs) located 3' of the T-cell leukemia 1A (TCL1A) gene that were associated with musculoskeletal adverse events induced by aromatase inhibitors (AIs) (Ingle et al., 2010). The top hit SNP (rs11849538) from that GWAS was in tight linkage disequilibrium with two other SNPs, $\operatorname{rs} 7160302$ and $\operatorname{rs} 7359033\left(R^{2}=0.92\right.$ and 0.98 , respectively) (Ho et al., 2016a). Subsequently, we performed functional genomic studies using a human variation panel of lymphoblastoid cell lines (LCLs), and we found that

This work was supported in part by the National Institutes of Health National Institute of General Medical Sciences [U19 GM61388 and R01 GM28157] and National Cancer Institute [P50 CA11620, R01 CA196648, and R01 CA138461]; the Avon Breast Cancer Crusade, the Breast Cancer Research Foundation, and the Mayo Clinic Center for Individualized Medicine. R.M.W. and L.W. are cofounders and stockholders in OneOme.

https://doi.org/10.1124/jpet.118.247718.

S This article has supplemental material available at jpet.aspetjournals.org.
TCL1A expression was induced by estradiol $\left(\mathrm{E}_{2}\right)$, but only in cell lines homozygous for variant genotypes for the TCL1A SNPs (Ho et al., 2016a). These three SNPs appeared to act in concert to influence estrogen-dependent TCL1A induction (Ho et al., 2016a). However, this expression pattern could be "reversed" after estrogen receptor $\alpha$ blockade with the active tamoxifen metabolite 4-hydroxytamoxifen (4OH-TAM).

We have also reported that mechanisms underlying this drug-induced reversal of TCL1A expression are due, at least in part, to altered estrogen receptor binding to estrogen response elements, two of which are at a distance from the SNPs (Ho et al., 2016a). Even more striking, a series of downstream immune mediator genes, including those encoding cytokines (Liu et al., 2012), chemokines (Ho et al., 2016a), and Toll-like receptors (TLRs) (Ho et al., 2017), responded in parallel with TCL1A SNP- and estrogen-dependent transcription. These observations have potential implications for the treatment of inflammatory diseases such as rheumatoid arthritis because their drug treatment targets these immune mediators. If the

ABBREVIATIONS: Al, aromatase inhibitor; bp, base pair; ChIP, chromatin immunoprecipitation; ChIP-seq, chromatin immunoprecipitation sequencing; DAPI, 2-(4-amidinophenyl)-1H-indole-6-carboxamidine; $\mathrm{E}_{2}$, estradiol; EMSA, electrophoretic mobility shift assay; FBS, fetal bovine serum; GWAS, genome-wide association study; IP, immunoprecipitation; kb; kilobase; LCL, lymphoblastoid cell line; NF- $\kappa \mathrm{B}$, nuclear factor $\kappa \mathrm{B}$; 4OH-TAM, 4-hydroxytamoxifen; RA, rheumatoid arthritis; PCR, polymerase chain reaction; RNA-seq, RNA sequencing; siRNA, small interfering RNA; SNP, single-nucleotide polymorphism; TCL1A, T-cell leukemia 1A; TLR, Toll-like receptor. 
TCL1A SNP genotypes were known, these observations raise the possibility that immune mediator gene expression could be manipulated by the use of drugs such as 4OH-TAM.

The molecular mechanisms underlying the effect of the TCL1A SNPs that result in changes in TCL1A expression in an estrogen- and 4OH-TAM-dependent fashion are not clear. The molecular mechanisms by which TCL1A expression might be associated with AI-induced musculoskeletal adverse events also remain unclear. Our study was designed to explore those molecular mechanisms. Specifically, we set out to determine whether TCL1A might function as a transcription factor that acts broadly - genome-wide - in a SNP- and estrogen-dependent fashion, and to study underlying mechanisms responsible for this genomic phenomenon using a human variation panel LCL model system.

We should emphasize that this LCL panel represents a genomic data-rich cell line model system that has repeatedly demonstrated its utility in the generation and testing of pharmacogenomic hypotheses (Ingle et al., 2010, 2013, 2016; Liu et al., 2012; Ho et al., 2016a, 2017). Specifically, it allowed us to select LCLs for study with any common genotype or combinations of genotypes, as demonstrated by the experiments described subsequently.

\section{Materials and Methods}

\section{Human Variation Panel Lymphoblastoid Cell Lines}

The human variation panel LCL model system consists of $300 \mathrm{LCLs}$ from healthy subjects of three ethnicities (100 European American, 100 African American, and 100 Han Chinese American). This LCL cell line model system was used to perform many of the experiments described in this report. This cell line model system provides genomewide mRNA expression as determined by Affymetrix (Santa Clara, CA) U133 2.0 Plus GeneChip expression arrays. SNP data were generated by the Illumina 550K and 510S SNP BeadChip SNP array (Illumina, San Diego, CA), and genotyping data were then used to impute approximately 7 million SNPs per cell line (Liu et al., 2012).

\section{RNA Sequencing and Data Analysis}

RNA was isolated from LCLs with known TCL1A SNP genotypes (two LCLs with homozygous wild-type and three LCLs with homozygous variant genotypes for the TCL1A SNPs). RNA sequencing (RNAseq) experiments were conducted by the Mayo Clinic Center for Individualized Medicine Medical Genomics Facility. RNA-seq libraries were prepared with the Ovation RNA-seq system v2 kit (NuGEN, San Carlos, CA) according to the manufacturer's instructions, and they were sequenced using an Illumina HiSeq 2000 with six samples in each lane using 100-base pair (bp) paired-end index reads. Fastq files containing paired RNA-seq reads were aligned with Tophat 2.0.12 (Kim et al., 2013) against the University of California-Santa Cruz (UCSC) human reference genome (hg19) using Bowtie 2.2.3 with default settings (Langmead and Salzberg, 2012). Gene level counts from uniquely mapped, nondiscordant read pairs were obtained using the subRead featureCounts program (v1.4.6) (Liao et al., 2013) and gene models from the UCSC hg19 Illumina iGenomes annotation package. Size factors were computed using the estimateSizeFactors function of DESeq2, and reference genes (GAPDH, ACTB) as the controlGenes parameter for estimateSizeFactors by the DESeq2 package (Love et al., 2014). Differential expression analysis was also performed using the DESeq2 package with default parameters.

\section{Identification of Genes with TCL1A Expression Patterns}

To identify genes with expression patterns similar to that of TCL1A after drug or hormone treatment, we mined our transcriptome data to identify genes that were up-regulated with $\log 2 \mathrm{FC}>1$ only in LCLs homozygous for wild-type sequences for the TCL1A SNPs in response to $\mathrm{E}_{2}$ treatment, and LCLs homozygous for variant genotypes for the TCL1A SNPs with nonsignificant changes or down-regulated $(\log 2 \mathrm{FC}<0.5)$ expression in response to $\mathrm{E}_{2}$ treatment. We also searched for genes that had "reversed" gene expression patterns in response to $\mathrm{E}_{2}+4 \mathrm{OH}-\mathrm{TAM}$ treatment. Specifically, these were genes that were up-regulated with $\log 2 \mathrm{FC}>1$ only in LCLs with homozygous variant sequences for the TCL1A SNPs in response to $\mathrm{E}_{2}+4 \mathrm{OH}-\mathrm{TAM}$ treatment, and LCLs with homozygous wild-type genotypes for the TCL1A SNPs that displayed nonsignificant changes or down-regulation $(\log 2 \mathrm{FC}<0.5)$ in response to $\mathrm{E}_{2}+4 \mathrm{OH}-\mathrm{TAM}$ treatment. Gene ontology terms for each data set were generated using Database for Annotation, Visualization and Integrated Discovery (DAVID) v6.8 (https://david. ncifcrf.gov/) (Huang et al., 2009).

\section{Chromatin Immunoprecipitation Sequencing and Data Analysis}

The chromatin immunoprecipitation sequencing (ChIP-seq) experiments were performed in duplicate using two LCLs with known TCL1A genotypes. Specifically, we selected one LCL from each genotype group from the five LCLs we used to perform RNA-seq. The ChIP-seq libraries were prepared using the Ovation ultralow DR Multiplex kit (NuGEN), and were subsequently sequenced to 51-bp paired ends using Illumina HiSeq 2000 at the Mayo Clinic Center for Individualized Medicine Medical Genomics Facility. The Fastq files were aligned against the UCSC human reference genome (hg19) with Bowtie 1.1.0 using the following Bowtie parameters: - sam -chunkmbs $512-\mathrm{p} 4-\mathrm{k} 1-\mathrm{m} 1-\mathrm{e}$ $70-151$-best. We then used the HOMER function findPeaks with default parameters to identify significantly enriched peaks in the experimental samples (Heinz et al., 2010).

We used deepTools to visualize binding profiles for TCL1A around transcription start sites ( $-2 \mathrm{~kb},+2 \mathrm{~kb}$ ) (Ramírez et al., 2016). Briefly, sorted bam files were used as input for the deepTools bamCoverage function using the parameter - normalizeTo1x 2451960000 for normalization and generating BigWig files. We then used the function computeMatrix with binSize $=20$ to compute scores for genome regions, and we used its output to plot a heat map of TCL1A binding profiles across vehicle- and drug-treatment conditions using the deepTools function plotHeatmap. To identify regions with distinct binding intensities, we clustered binding profiles into four clusters using k-means.

Next, we used the ChipSeeker package to annotate peaks identified by HOMER and study the distribution of read densities around annotated genomic regions. Motif analyses were performed using the HOMER function findMotifsGenome with the parameter size $50-$ mask to identify transcription factor-binding motifs. The accession number for the p65 ChIP-seq data are GSE55105 (Zhao et al., 2014).

Overlap analysis for genome-wide TCL1A and p65 binding sites was performed using a window of $\pm 500 \mathrm{bp}$ from the center of each peak of TCL1A binding, and the binding signals of TCL1A and p65 were plotted side by side for each window, as shown in Fig. 5B. Figure 5C sums the ChIP-seq signals for TCL1A and p65 bindings, respectively, using a window $\pm 1000 \mathrm{bp}$ from the transcription start site from each of the 357 TCL1A target genes we studied. Correlations were then calculated between TCL1A and p65 binding for each treatment condition.

CRISPR/Cas9 Plasmids and Knockout TCL1A Gene. TCL1A Double Nickase Plasmid sc-402028-NIC (Santa Cruz Biotechnology, Dallas, TX) was transfected into LCLs using the program X-001 of Amaxa Nucleofector (Lonza, Köln, Germany), and re-covered for 24 hours in RPMI1640 medium (Cellgro, Manassas, VA) supplemented with $15 \%$ fetal bovine serum (FBS; Atlanta Biologicals, Flowery Branch, GA). Transfected cells were cultured in medium containing $0.2 \mu \mathrm{g} / \mathrm{ml}$ of puromycin for 10 days for selection. Cells were seeded on a 96-well culture plate using RPMI1640 medium 
supplemented with $15 \%$ FBS for 12 weeks. TCL1A expression was quantified and normalized to the $\beta$-actin signal by Western blot analysis. Cells exhibiting $<10 \%$ normalized TCL1A signal were considered to have TCL1A knocked out.

\section{Drug Treatment}

LCLs with known genotypes were cultured in RPMI 1640 medium (Cellgro) supplemented with 15\% FBS (Atlanta Biologicals). Before estrogen treatment, the cells were cultured in RPMI media containing $5 \%(\mathrm{v} / \mathrm{v})$ charcoal-stripped FBS for 24 hours, and were subsequently cultured in FBS-free RPMI medium for another 24 hours. The cells were then treated with $0.1 \mathrm{nM}$ of $\mathrm{E}_{2}$ for 24 hours. In some experiments, the cells were treated with $4 \mathrm{OH}-\mathrm{TAM}\left(10^{-7} \mu \mathrm{M}\right)$.

\section{Real-Time Polymerase Chain Reaction}

Four cell lines with each TCL1A genotype were selected for functional validation. The polymerase chain reaction (PCR) mixture contained $100 \mathrm{ng}$ of total RNA, $5 \mu \mathrm{l}$ of $2 \mathrm{X}$ VeriQuest SYBR green $\mathrm{qPCR}$ master mix (Affymetrix), $0.1 \mu \mathrm{l}$ of DNA polymerase, $1 \mu \mathrm{l}$ of genespecific primer, and distilled water up to $10 \mu \mathrm{l}$ per reaction. GAPDH and ACTB were used as internal controls. Real-time PCR reactions were performed in duplicate using the ViiA 7 Real-Time PCR System (Applied Biosystems/Life Technologies, Carlsbad, CA). The $2^{-\Delta \Delta \mathrm{Ct}}$ method was employed for statistical data analysis.

\section{RNA Interference and Transfection}

Pooled TCL1A, p65 siRNA, and negative control were purchased from Dharmacon (Chicago, IL). Small interfering RNA (siRNA) was transfected into the LCLs by electroporation using the nucleofector kit (Lonza). Briefly, the electroporation reaction contained $2.5 \times 10^{6}$ cells, $100 \mu \mathrm{l}$ of nucleofector solution, and $300 \mathrm{nM}$ siRNA. After electroporation, the cells were transferred into 12 -well plates containing preequilibrated RPMI medium for use in the experiments described in this report.

\section{Chromatin Immunoprecipitation Assay and ChIP-re-ChIP Assay}

ChIP assays were performed using LCLs with known TCL1A SNP genotypes ( $n=4$ for each genotype) using the EpiTect ChIP OneDay Kit (Qiagen, Valencia, CA). DNA-TCL1A complexes were immunoprecipitated using antibodies against TCL1A, RelA, or with normal mouse IgG (Cell Signaling Technology, Danvers, MA) as a control. ChIP-re-ChIP assays were performed using the Re-ChIP-IT Kit (Active Motif, Carlsbad, CA). Real-time PCR was used to quantify TCL1A binding or p65 binding. The primer sets for the ChIP assays are listed in Supplemental Table 4.

\section{Coimmunoprecipitation of TCL1A and Nuclear Factor $\kappa$ B}

The LCLs $\left(1 \times 10^{7}\right)$ were resuspended in $650 \mu$ l of immunoprecipitation (IP) lysis buffer containing $2.2 \mu \mathrm{l}$ of protease inhibitor cocktail (Qiagen) and were incubated on ice for 30 minutes. The cells were then centrifuged at $12,000 \mathrm{~g}$ at $4^{\circ} \mathrm{C}$ for 15 minutes. The supernatants were collected. Protein A agarose (ThermoScientific, Madison, WI) was prepared and washed 3 times with IP lysis buffer. A precleaning step was performed to clean the background. Cell lysates containing protein A agarose beads were rotated at $4^{\circ} \mathrm{C}$ for an hour. After centrifugation, the supernatant was collected. At this point, input $(50 \mu \mathrm{l})$ was collected and stored at $-80^{\circ} \mathrm{C}$.

Anti-TCL1A (1:50) (Cell Signaling Technology) or anti-nuclear factor $\kappa \mathrm{B}$ (anti-NF- $\kappa \mathrm{B})$ p65 (1:50) antibodies (Abcam, Cambridge, MA) were used to perform IP. IgG (Cell Signaling Technology) was used as the negative control. Specifically, IP samples containing protein $\mathrm{A}$ agarose beads were rotated at $4^{\circ} \mathrm{C}$ overnight. Immunoprecipitates were washed 3 times with ice-cold lysis buffer, and the proteins were eluted with $50 \mu \mathrm{l}$ of $1 \mathrm{X}$ Laemmli loading buffer. The proteins were separated on 4\%-12\% SDS-PAGE gels and transferred onto polyvinylidene fluoride membranes. After blocking, membranes were incubated with primary antibodies against TCL1A or NF- $\kappa \mathrm{B}$ p65 at $4^{\circ} \mathrm{C}$ overnight. The washed membrane was then incubated with secondary antibody (1:15,000 dilution) for an hour at room temperature. The membrane was visualized using super signal ECL substrate (ThermoScientific).

\section{Electrophoretic Mobility Shift Assay}

Recombinant Human TCL1A Protein (NBP1-30239) was purchased from Novus Biologicals (Littleton, CO). Synthetic oligonucleotides (sense and antisense) were derived from TCL1A response element sequences. Double-stranded DNA probes were $3^{\prime}$ end-labeled with biotin (IDT, Coralville, IA). A 100-fold excess of unlabeled oligonucleotide competitors was used for the competition experiments. The reaction mixture was then loaded onto a 5\% Tris/Borate/EDTA native polyacrylamide gel and electrophoresis was run for 1 hour at $100 \mathrm{~V}$ in $0.5 \times$ Tris/Borate/EDTA buffer. The protein-DNA complexes were transferred to Biodyne B Nylon Membranes (ThermoScientific) and UV cross-linked. An electrophoretic mobility shift assay (EMSA) was performed using LightShift Chemiluminescent EMSA Kit (ThermoScientific).

\section{Immunofluorescence Staining and Confocal Imaging Analysis}

LCLs were grown on glass coverslips and treated with $0.1 \mathrm{nM} \mathrm{E}_{2}$ for 24 hours. The cells were then fixed in $4 \%$ paraformaldehyde at room temperature for 10 minutes. The cells were washed in cold phosphatebuffered saline and permeabilized with $0.2 \%$ Triton X-100. After blocking for 1 hour with $3 \%$ bovine serum albumin, the cells were incubated with mouse anti-TCL1A antibody (Cell Signaling Technology) overnight at $4^{\circ} \mathrm{C}$. The secondary antibody (red) was ab150119 Alexa Fluor 647 goat anti-mouse $\operatorname{IgG}(\mathrm{H}+\mathrm{L})$ used at 1:1000 dilution for an hour. DAPI [2-(4-amidinophenyl)- $1 H$-indole-6-carboxamidine] was used to stain the cell nuclei (blue) at a concentration of $1.43 \mu \mathrm{M}$. The slides were visualized using fluorescence microscopy (FV1200; Olympus, Tokyo, Japan).

\section{Results}

RNA-Seq Genome-wide Identification of TCL1A SNPand Estrogen-Dependent Transcription Regulation. As a first step, RNA-seq was performed using LCLs selected for either homozygous wild-type or homozygous variant genotypes for the TCL1A SNPs shown graphically in Fig. 1A. The cells were treated with vehicle, $\mathrm{E}_{2}$, or $\mathrm{E}_{2}+4 \mathrm{OH}$-TAM. The drug concentrations used had been optimized as shown in Supplemental Fig. 1 (Liu et al., 2012; Ho et al., 2016a, 2017). We then randomly selected 39 genes for use in the validation of the RNA-seq data and found very high reproducibility based on the use of qPCR (Supplemental Fig. 2). For example, in the presence of $\mathrm{E}_{2}$, TCL1A expression was induced in LCLs that were homozygous variant but not in those with homozygous wild-type genotypes for the TCL1A SNPs (Fig. 1B).

As expected, this expression pattern could be reversed in the presence of 4OH-TAM. Specifically, TCL1A expression was significantly up-regulated in cells that were homozygous wildtype for the TCL1A SNPs but not in those with homozygous variant genotypes (Fig. 1B). Based on the RNA-seq data, 357 genes displayed this TCL1A SNP- and estrogen-dependent gene expression pattern-a pattern that could be reversed by $4 \mathrm{OH}-$ TAM (Fig. 1C), in parallel with the pattern of expression of TCL1A itself in response to treatment with either $\mathrm{E}_{2}$ or $\mathrm{E}_{2}+$ 4OH-TAM (Fig. 1B). 


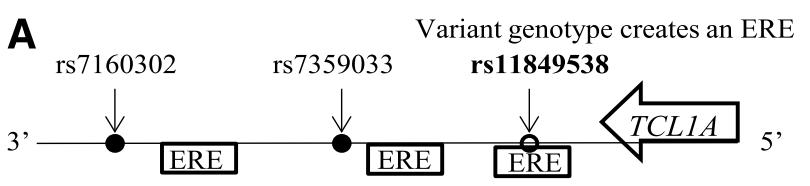

B
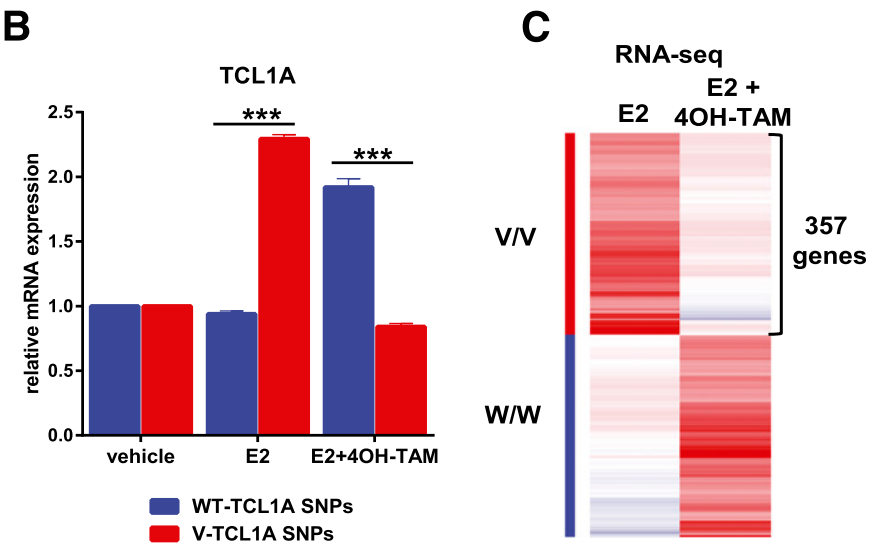

Fig. 1. (A) Schematic diagram of three TCL1A SNPs: rs11849538, the "top hit" signal from the MA.27 musculoskeletal adverse event GWAS, rs7359033, and rs7160302. All three of these SNPs map near the $3^{\prime}$ terminus of TCL1A and are in tight linkage disequilibrium. Locations of estrogen response elements (EREs) are shown as boxes. (B) SNP- and estrogen-related variation of TCL1A mRNA expression in lymphoblastoid cell lines with known TCL1A SNP genotypes after exposure to $\mathrm{E}_{2}$ with or without 4OH-TAM. Student's $t$ test was performed to compare gene expression in LCLs with differing TCL1A SNP genotypes for each treatment condition, $* * * P<0.001$. (C) Heat map showing expression profiles for 357 genes regulated by TCL1A SNPs in an estrogen-dependent fashion, all of which could be reversed by $4 \mathrm{OH}-\mathrm{TAM}$ treatment, as determined by RNA-seq using LCLs with either homozygous wild-type $(\mathrm{W} / \mathrm{W})(n=2)$ or homozygous variant $(\mathrm{V} / \mathrm{V})(n=3)$ genotypes for the TCL1A SNPs.

Specifically, a total of 357 genes could be significantly induced by $\mathrm{E}_{2}$ with $\log 2$-fold change $\geq 1$ (Supplemental Table 1), but only in LCLs homozygous for TCL1A SNP variant genotypes (Fig. 1C). However, the expression of those same 357 genes was significantly induced in the presence of 4OH-TAM together with $\mathrm{E}_{2}$ in LCLs that were homozygous for wild-type genotypes for the TCL1A SNPs (Fig. 1C).

We also performed gene ontology analysis and found that these 357 genes clustered within pathways involved in transcriptional regulation and T-cell activation (Supplemental Table 2) by using the Database for Annotation, Visualization and Integrated Discovery (DAVID) v6.8 (https://david. ncifcrf.gov/) (Huang et al., 2009). These results provided additional information with regard to the role of TCL1A in the transcriptional regulation of immune mediators (Liu et al., 2012; Ho et al., 2016a,b). In the next series of experiments, we set out to directly test by ChIP-seq the possibility that TCL1A was a novel transcription factor that acts in a SNP- and estrogen-dependent fashion.

ChIP-Seq Genome-wide Identification of TCL1A Binding Sites. We next examined TCL1A's binding profile on a genome-wide scale by performing ChIP-seq using the same set of LCLs that had been used in the RNA-seq studies. We clustered Chip-seq peak signals within $\pm 2 \mathrm{~kb}$ from the transcription start site into four clusters using K-means. We observed that genes in cluster 1 were more strongly bound by TCL1A than genes in clusters 2 and 3, with higher enrichments in variant genotypes after $\mathrm{E}_{2}$ treatment (Fig. 2A).
Therefore, TCL1A was found to bind preferentially to promoter regions. In addition, TCL1A also bound significantly in introns ( $\sim 30 \%$ of sites) and intergenic regions ( $23 \%)$ (Fig. 2B).

We also performed ChIP-qPCR experiments for 25 randomly selected sites using independent samples to confirm the specificity of these observations (Supplemental Fig. 3). The most significantly associated gene ontology terms for these genes involved pathways related to transcription regulation and immune function (Supplemental Table 3). This result confirmed our previous findings with regard to the effect of TCL1A on the expression of cytokines, chemokines, and their receptors (Supplemental Fig. 1), all of which play an important role in the regulation of immune responses. Our ChIP-seq results showed that TCL1A is capable of binding to specific DNA sequences across the human genome, resulting in the regulation of the transcription of target genes.

TCL1A Response Elements. To identify in an unbiased fashion sequence motifs at sites occupied by TCL1A, we examined DNA sequences (50 bp \pm from the peak) using HOMER (http://homer.salk.edu/homer/ngs/) (Heinz et al., 2010) and MEME (Bailey et al., 2009). De novo motif search in each set of TCL1A binding sites from these samples revealed enrichment of the consensus sequence 5'-CCATATATGG-3', which we designated as a TCL1A consensus DNA binding motif (Fig. 2C). We then performed EMSA using biotin-labeled TCL1A binding motif sequences and recombinant TCL1A protein to confirm that TCL1A protein could recognize and bind to this DNA motif sequence (Fig. 3A). The next series of studies was designed to characterize TCL1A as a novel transcription factor, with effects that occur with and without 4OH-TAM exposure.

Estrogen Significantly Alters TCL1A Occupancy of Binding Sites in or near Target Genes in a SNP-Dependent Fashion. In the absence of $E_{2}$, TCL1A is mainly expressed in the cytoplasm and, to a lesser degree, in the cell membrane as determined by immunofluorescence staining of LCLs (Fig. 3B). However, TCL1A migrates and accumulates in the nucleus in the presence of $\mathrm{E}_{2}$ (Fig. 3B). This observation is important because it supports the ability of TCL1A to translocate into the nucleus where it can function as a transcription factor, with effects that can be altered by estrogens and by the SNPs located 3' of TCL1A. For example, ChIP-seq showed that TCL1A was capable of binding to sites in the CCR6 and IL17RA genes, both of which can be regulated by TCL1A in a SNP- and estrogen-dependent fashion (Ho et al., 2016a) (Fig. 3C).

Even more striking, the binding density depended on TCL1A SNP genotype and $\mathrm{E}_{2}$ treatment (Fig. 3C). Specifically, TCL1A binding density in peaks in both CCR6 and IL17RA was significantly higher in cells with homozygous variant genotypes for the TCL1A SNPs as compared with results for cells with homozygous wild-type genotypes. These results parallel those found during the RNA-seq studies (Fig. 3D).

It should be emphasized that the SNPs were located $3^{\prime}$ of the TCL1A gene but not in CCR6 or IL17RA. These observations led us to test whether the SNP- and estrogen-dependent expression of TCL1A might be associated with the transcription of TCL1A target genes. Therefore, the next series of studies was designed to integrate the ChIP-seq and RNA-seq data and to explore the possible role of 


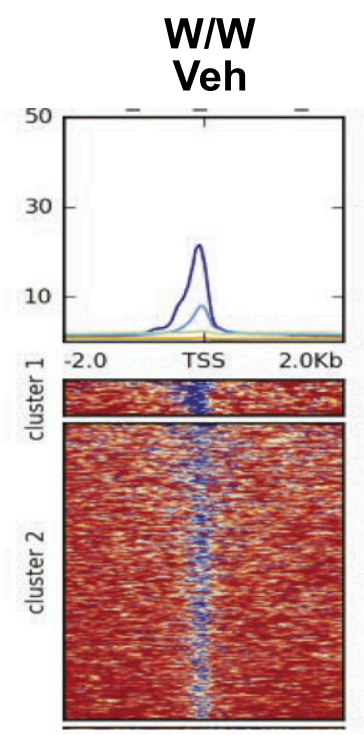

-2kb TSS $2 k b$
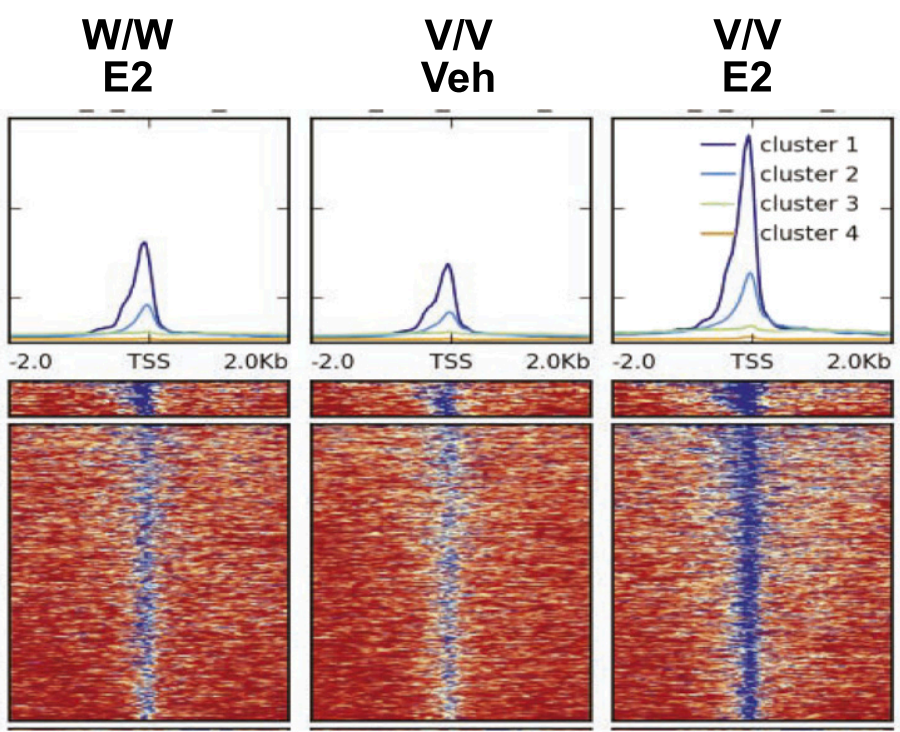

$-2 k b$ TSS $2 k b$

$-2 k b$ TSS $2 k b$

B

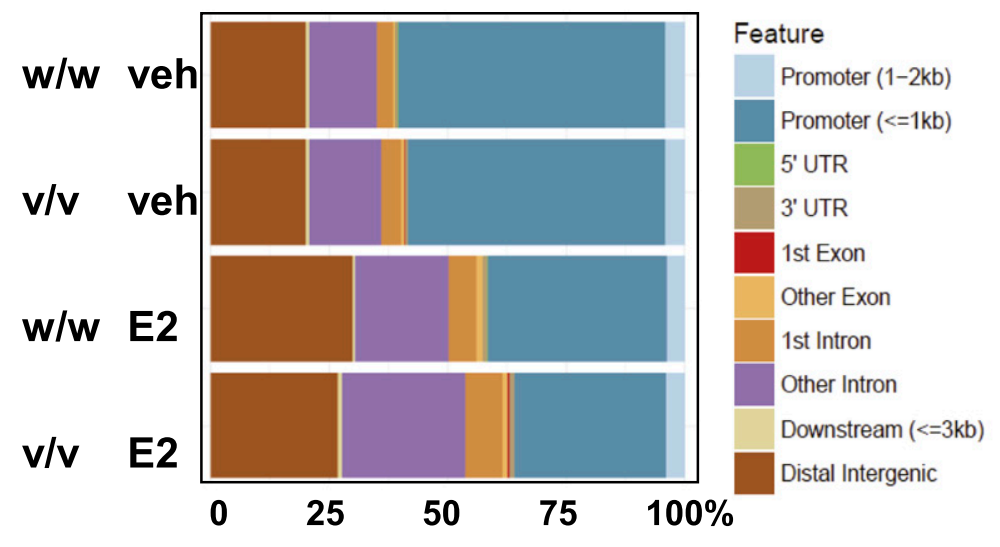

C

\begin{tabular}{|c|c|c|c|}
\hline \multirow{2}{*}{$\begin{array}{l}\text { TCL1A SNP } \\
\text { genotypes } \\
\text { W/W }\end{array}$} & \multicolumn{3}{|c|}{ HOMER } \\
\hline & $\begin{array}{l}\text { Treatment } \\
\text { Vehicle }\end{array}$ & $\begin{array}{l}\text { p value } \\
p=1 e-166\end{array}$ & $\begin{array}{l}\text { motif sequence } \\
\text { CCATATATGG }\end{array}$ \\
\hline w/w & E2 & $p=1 e-179$ & 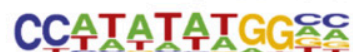 \\
\hline v/v & Vehicle & $p=1 e-495$ & CCATATATGG웃도 \\
\hline $\mathrm{v} / \mathrm{v}$ & E2 & $\mathrm{p}=1 \mathrm{e}-206$ & CCWATATATGGGE \\
\hline TCL1A SNP & & MEME & \\
\hline $\begin{array}{l}\text { genotypes } \\
\text { W/w }\end{array}$ & $\begin{array}{l}\text { Treatment } \\
\text { Vehicle }\end{array}$ & $\begin{array}{l}p \text { value } \\
p=1 e-158\end{array}$ & 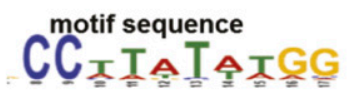 \\
\hline w/w & E2 & $p=1 e-150$ & 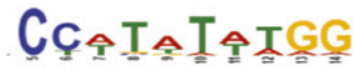 \\
\hline $\mathrm{v} / \mathrm{v}$ & Vehicle & $p=1 e-084$ & CC \\
\hline v/v & E2 & $p=1 e-117$ & 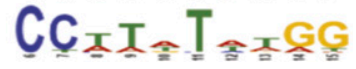 \\
\hline
\end{tabular}

Fig. 2. (A) Genome-wide TCL1A occupancy profiles. Regions (Tss $\pm 2 \mathrm{~kb}$ ) were clustered based on their profiles for TCL1A ChIP enrichment over input for all four samples using k-means clustering. Clustering was used to identify regions with distinct binding intensities. The gradient blue-to-red color indicates high-to-low counts in the corresponding genome region. (B) Percentage distributions of TCL1A ChIP-seq peaks for all four samples. (C) Identification of TCL1A consensus binding sequence motifs using HOMER and MEME.

TCL1A in transcriptional regulation in a SNP- and estrogendependent manner.

Integrated ChIP-Seq and RNA-Seq Analyses Reveal Functional TCL1A Binding Sites Associated with Transcriptional Regulation in a SNP-and EstrogenDependent Fashion. To explore the consequences of SNPdependent TCL1A binding in or near target genes in response to $\mathrm{E}_{2}$ treatment, we merged RNA-seq and ChIP-seq data by overlapping the results for genes that displayed TCL1A SNPdependent gene expression and SNP-dependent TCL1A binding in response to $\mathrm{E}_{2}$ treatment. A total of 357 genes could be induced by $\mathrm{E}_{2}$ in a TCL1A SNP-dependent fashion, and 57 of those genes also displayed binding of TCL1A with significantly increased binding density in response to $\mathrm{E}_{2}$ treatment but only in the cells with homozygous variant genotypes for the TCL1A SNP genotypes (Fig. 4A). We confirmed this observation by performing ChIP-qPCR with primers specifically designed to amplify genomic regions for TCL1A binding sites in these genes.

Specifically, for all 57 TCL1A binding regions, greater TCL1A binding was observed in the presence of $\mathrm{E}_{2}$ in cells with homozygous variant genotypes for the TCL1A SNPs as compared with those with wild-type genotypes (Fig. 4B). Furthermore, in the presence of 4OH-TAM this TCL1A 
A

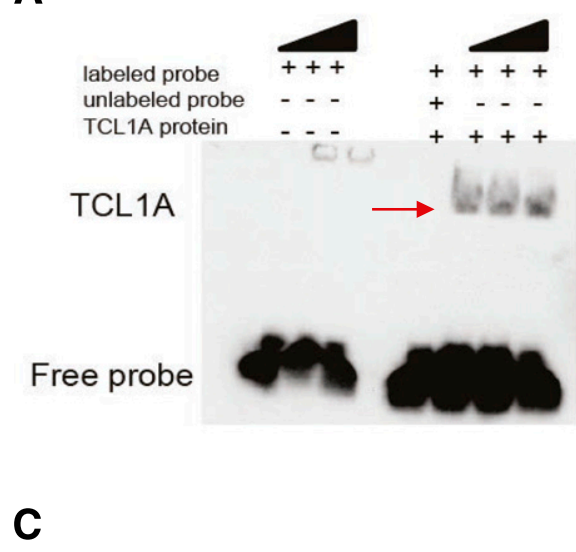

B

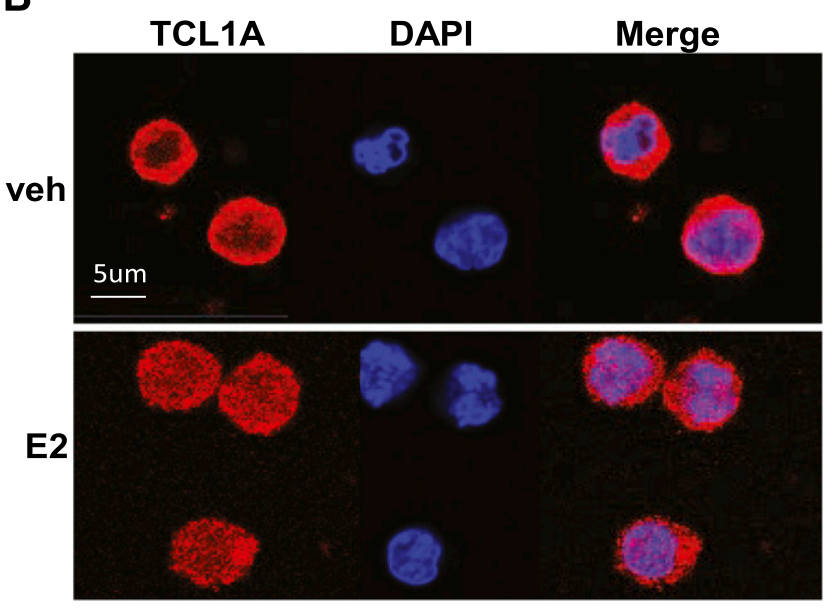

\section{TCL1A SNP \\ Genotypes Treatment}

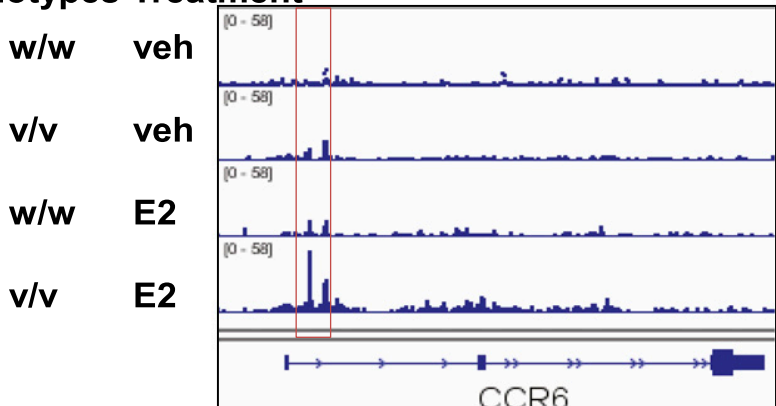

CCR6

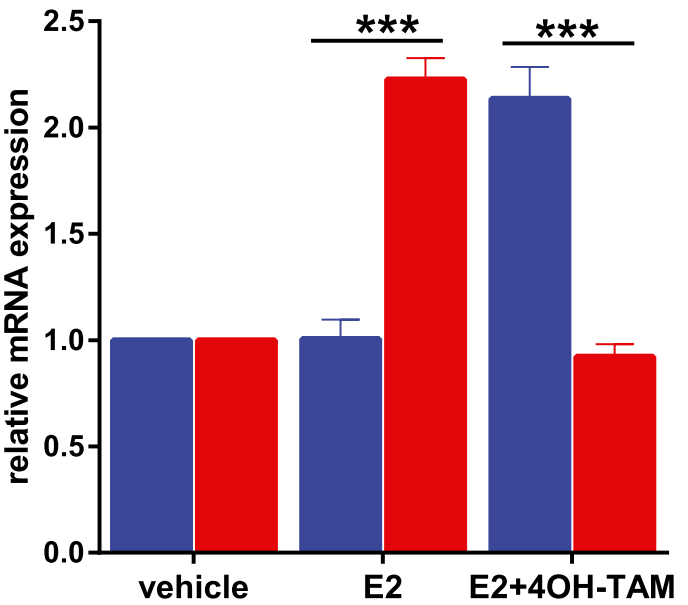

\section{WT-TCL1A SNPS \\ V-TCL1A SNPS}

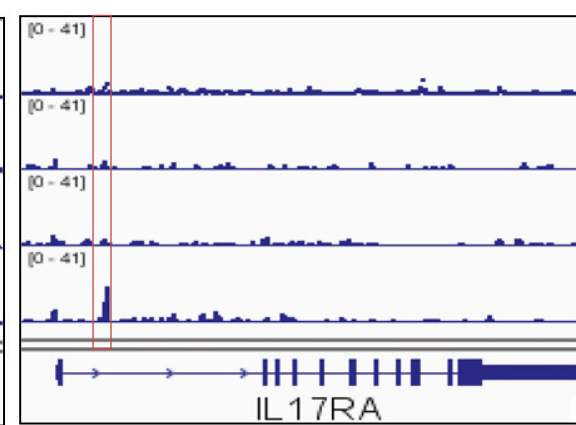

IL17RA

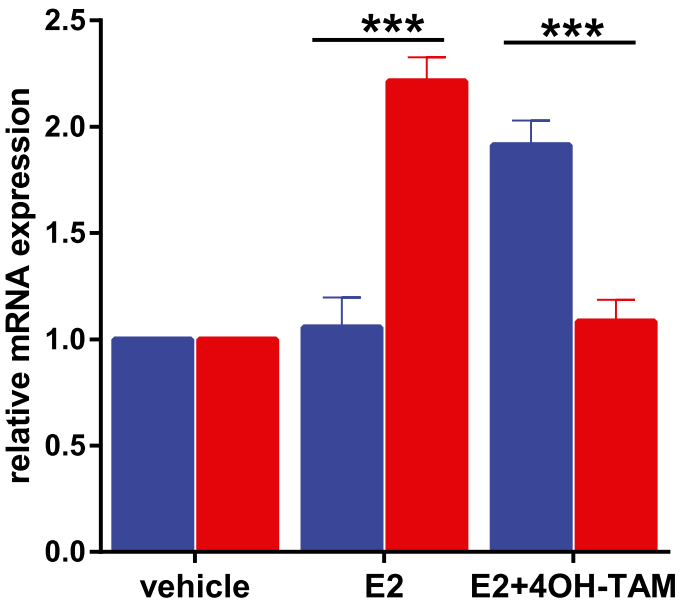

WT-TCL1A SNPS

\section{V-TCL1A SNPS}

Fig. 3. (A) EMSA results showing that one TCL1A DNA binding motif sequence, CCATATAGG, is sufficient for DNA-TCL1A protein interaction. (B) Immunofluorescence staining showing TCL1A nuclear translocation in response to $\mathrm{E}_{2}(0.1 \mathrm{nM})$ treatment in LCLs. (C) Representative examples of TCL1A occupancy peaks depicted for the CCR6 and IL17RA loci in LCLs with known TCL1A SNP genotypes in the absence of $\mathrm{E}_{2}$ or in the presence of $\mathrm{E}_{2}$ $(0.1 \mathrm{nM})$. Red boxes indicate that TCL1A binding in both CCR6 and IL17RA is significantly increased in response to $\mathrm{E}_{2}$ treatment, but that only occurs in cells with homozygous variant genotypes for TCL1A SNPs. (D) Changes in TCL1A occupancy are highly correlated with changes in mRNA expression levels for CCR6 and IL17RA. Student's $t$ test was performed to compare gene expression in LCLs with differing TCL1A SNPs (homozygous wild-type versus homozygous variant) for each treatment condition, $* * * P<0.001$.

binding pattern was reversed (Fig. 4C). These results were compatible with the gene expression patterns for all 57 of these genes in response to $\mathrm{E}_{2}$ treatment (Fig. 4D), all of which also displayed a reversal of expression in the presence of 4OH-TAM (Fig. 4E) using the same cell lines from which the data shown in Fig. 4, B and C, were obtained. It should be pointed out once again that the SNPs were located 3 ' of TCL1A and not in the 57 genes themselves. In summary, this series of 
A

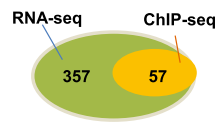

TCL1A ChIP assays: E2 treatment

B

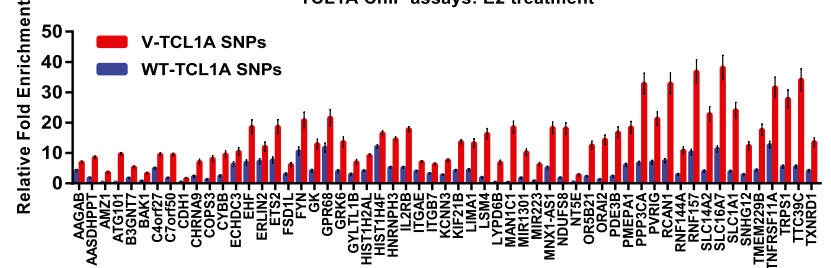

C

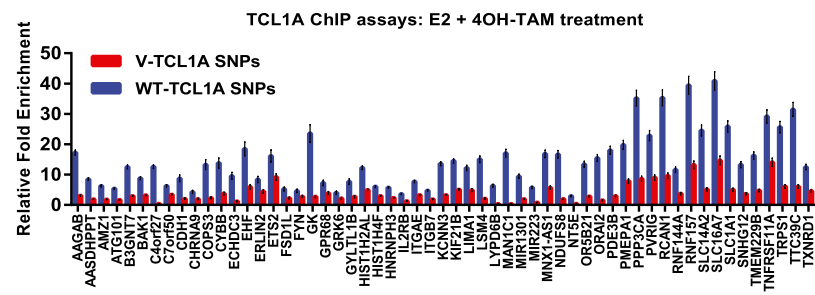

D

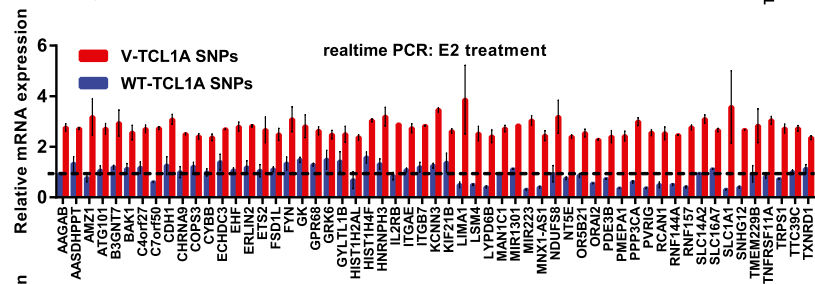

E

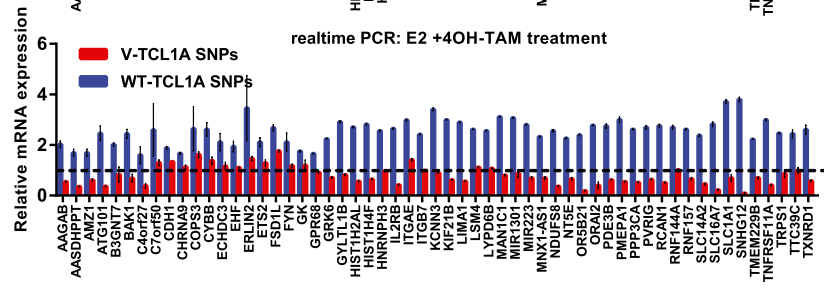

Fig. 4. (A) Venn diagram showing 357 gene that displayed TCL1A SNPand estrogen-dependent gene expression patterns as determined by RNA-seq, and 57 of those 357 genes that displayed TCL1A SNP- and estrogen-dependent TCL1A occupancy. (B and C) TCL1A ChIP assays were performed to confirm results obtained from TCL1A ChIP-seq for all 57 genes, all of which showed greater TCL1A binding in the presence of $\mathrm{E}_{2}$, but only in cells homozygous variant for the TCL1A SNP genotypes. In contrast, in the presence of $4 \mathrm{OH}-\mathrm{TAM}$, this binding pattern was reversed for all 57 genes ( $n=4$ for each genotype group). (D and $\mathrm{E}$ ) Changes in TCL1A binding were correlated with changes in mRNA expression for all 57 genes using the same cell lines from which the data shown in $B$ and $C$ were obtained. Specifically, in the presence of $E_{2}$, all those genes showed significant induction only for the variant genotype. However, the expression pattern could be reversed by $4 \mathrm{OH}-\mathrm{TAM}$, thus confirming that changes in TCL1A occupancy were highly correlated with transcription.

experiments demonstrated clearly that TCL1A served as a transcription factor for these target genes in a SNP- and estrogen-dependent fashion.

TCL1A and NF-кB p65 Co-occupy Binding Sites on TCL1A-Responsive Target Genes. We also interrogated the sequences of the TCL1A binding sites (peak $\pm 50 \mathrm{bp}$ ) for overrepresentation of known DNA binding motifs using HOMER. An NF-kB p65 binding motif was highly enriched in these genomic regions (with $P$ values $=1 \mathrm{E}-12$ and $1 \mathrm{E}-38$ for cells with homozygous wild-type or homozygous variant genotypes for the TCL1A SNPs, respectively). These observations led us to determine whether TCL1A might physically interact with $\mathrm{NF}-\kappa \mathrm{B}$ p65 and, as a result, might serve as a transcriptional coregulator with NF- $\kappa \mathrm{B}$ p65.
Possible protein-protein interaction between TCL1A and $\mathrm{NF}-\kappa \mathrm{B}$ p65 was determined by coimmunoprecipitation (co-IP) using LCLs with endogenous expression of both TCL1A and NF- $\kappa \mathrm{B}$ p65 (Fig. 5A). Reverse IP verified the physical association between NF- $\kappa \mathrm{B}$ p65 and TCL1A (Fig. 5A). We next tested whether both TCL1A and NF- $\kappa$ B p 65 might target the same genes and/or regulatory elements. To do that, we compared our TCL1A ChIP-seq dataset and publicly available p65 ChIP-seq dataset generated for LCLs (Zhao et al., 2014). There was a high degree of overlap between the locations of peaks for the two transcription factors (Fig. 5B).

It should be pointed out that the heat map for p65 binding sites shown in Fig. $5 \mathrm{~B}$ used a window \pm 500 bp centered on TCL1A peaks genome-wide, which indicated that $\mathrm{p} 65$ bound to genome regions similar to those to which TCL1A bound. This overlap suggested a possible functional relationship between TCL1A and p65 in the regulation of gene expression, including genes involved in immune response. This overlap was particularly striking for the 357 genes shown in Fig. 1C, genes that could be regulated by TCL1A in a SNP- and estrogendependent manner (Fig. 5C). Therefore, we next attempted to determine whether TCL1A and the NF- $\kappa \mathrm{B}$ p65 subunit might interact within the same transcriptional complex by performing ChIP-re-ChIP assay.

Those experiments showed that TCL1A and NF- $\kappa \mathrm{B}$ p65 co-occupied the same sites on TCL1A target genes, as shown in Fig. 6, A and B, based on the results of ChIP-re-ChIP assays performed using antibody against TCL1A, followed by antibody against NF- $\kappa \mathrm{B}$ p65. Even more striking, the binding pattern for NF- $\kappa \mathrm{B}$ p65 displayed a TCL1A SNP- and estrogendependent pattern. Specifically, a significant increase in NF- $\kappa$ B p65 binding was observed in cells with homozygous variant genotypes for the TCL1A SNPs in response to $\mathrm{E}_{2}$ treatment (Fig. 6A). However, this binding pattern was reversed when the cells were exposed to 4OH-TAM (Fig. 6B), in parallel with the binding pattern for TCL1A that we had observed (Fig. 4, B and C). It should be emphasized once again that the SNPs being studied were located $3^{\prime}$ of TCL1A not in the NF- $\kappa \mathrm{B}$ p65 gene or in the genes listed in Fig. 6, A and B.

TCL1A and p65 expression displayed a positive correlation in LCLs (Fig. 7A). Specifically, TCL1A knockout in LCLs using CRISPR-Cas9 resulted in the down-regulation of p65. NF- $\kappa \mathrm{B}$ p65 knockout resulted in cell death, so we used siRNA transient knockdown of this gene in our experiments. Similarly, knockdown of p65 in LCLs using siRNA caused down-regulation of TCL1A (Fig. 7A). Furthermore, TCL1A SNP- and estrogen-dependent binding to DNA often appeared to involve both TCL1A and NF- $\kappa$ B p65. For example, the SNP-dependent binding pattern for TCL1A target genes was lost after the knockdown of p65 in LCLs with known TCL1A SNP genotypes (Fig. 7B). In parallel, the SNP- and estrogen-dependent p65 binding for TCL1A target genes was also abolished after TCL1A was knocked out (Fig. 7C). Furthermore, the TCL1A SNP- and estrogendependent gene expression pattern was also lost after p65 was knocked down (Fig. 7D) or TCL1A was knocked out (Fig. 7E).

Taken together, these data suggest that a TCL1A and $\mathrm{NF}-\kappa \mathrm{B}$ p65 protein complex might contribute to TCL1A SNP- and estrogen-dependent transcription regulation, at least for some genes. We also observed that TCL1A SNPand estrogen-dependent p65 binding was lost after TCL1A 


\section{A Co-Immunoprecipitation}

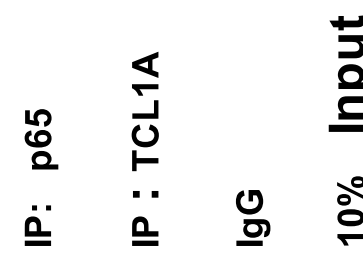

IB: p65

IB: TCL1A

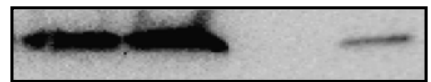

B

\section{ChIP-seq}

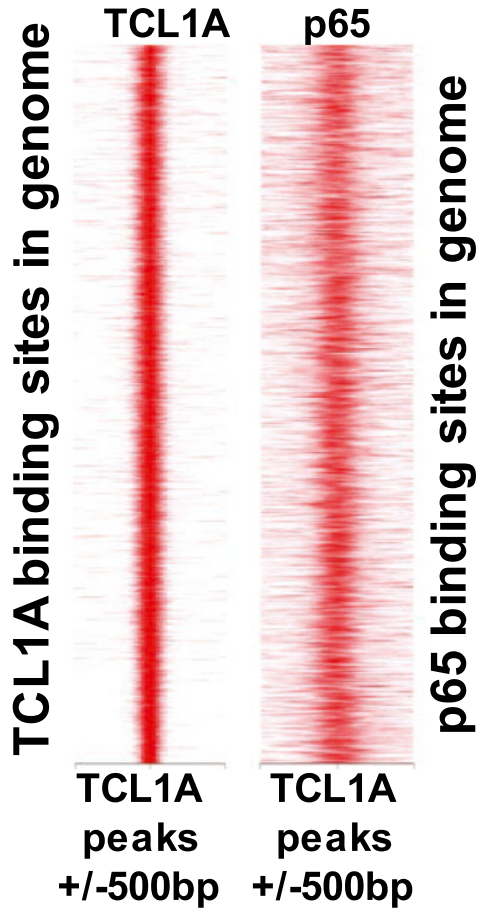

Fig. 5. (A) Coimmunoprecipitation was used to determine whether TCL1A protein could interact with p65 in LCLs. Whole cell lysates from $1 \times 10^{7}$ LCLs were immunoprecipitated with (left panel) anti-TCL1A (1: 50) antibodies or anti-IgG antibodies and protein samples were immunoblotted and probed with antibodies against TCL1A. Reversed IP was preformed to confirm that p65 and TCL1A interacted (right panel). (B) Heat-map plots showing the association between TCL1A binding and p65 binding in LCLs (Zhao et al., 2014). The signals for TCL1A ChIP-seq peaks and p65 ChIP-seq peaks are shown as heat-maps using red (the strongest signal) and white (the weakest signal) color schemes. Each row shows \pm 500 bp centered on the TCL1A ChIP-seq peak summits. (C) TCL1A occupancy versus p65 occupancy in LCLs. Scatter plots depicting the Pearson correlations ( $r$ ) between TCL1A ChIP tag density ( $y$ axis) and p65 ChIP tag density ( $x$ axis) for the 357 TCL1A target genes are shown in Fig. 1C.
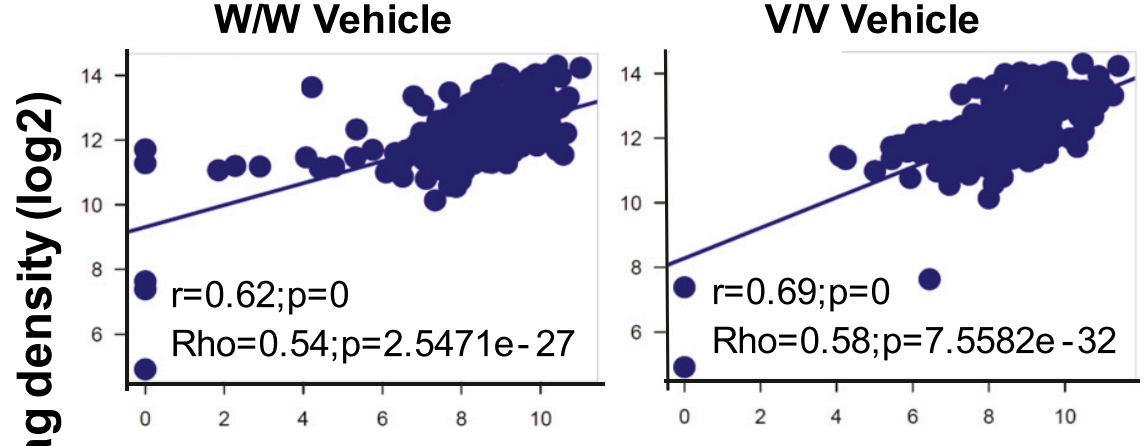

W/W E2

V/V E2

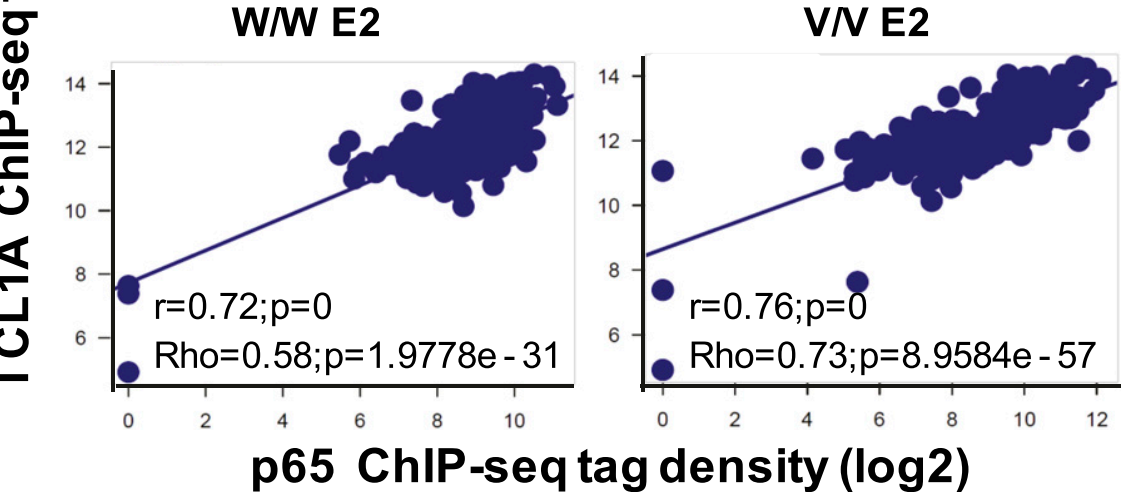

knockout (data not shown). These data suggested that TCL1A and NF- $\kappa$ B p65 either co-occupy the same sites or that their binding sites are located very close together on TCL1A target genes, resulting in the SNP- and estrogendependent variation in gene expression that we observed.
Finally, TCL1A also appeared to interact with all NF- $\kappa$ B subunits except for p52 (Fig. 8). However, relB, c-rel, and p50 were not able to bind the TCL1A target genes in areas of TCL1A binding (Supplemental Fig. 4). Taken as a whole, these results supported the conclusion that NF- $\kappa \mathrm{B}$ p65 may 

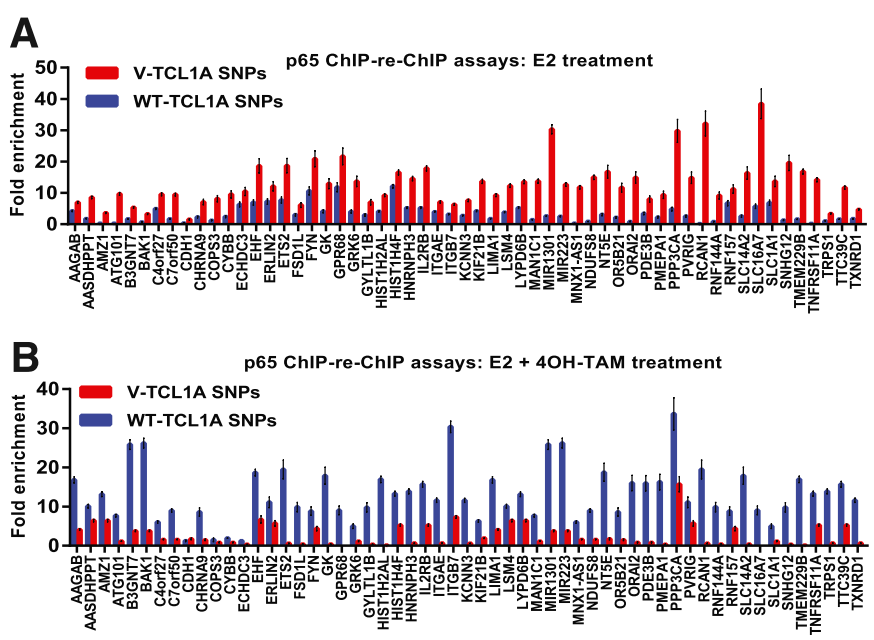

Fig. 6. (A and B) p65 ChIP-re-ChIP assays were performed to confirm the co-occupancy of TCL1A and p65 on 57 selected binding regions as shown in Fig. $4, \mathrm{~B}$ and $\mathrm{C}$ ( $n=4$ for each genotype group).

play an important role in TCL1A SNP- and estrogendependent transcriptional regulation.

\section{Discussion}

We have studied mechanisms responsible for TCL1A SNPand estrogen-dependent regulation of gene expression using a cell-line model system. TCL1A is expressed in immune cells including activated T lymphocytes, B lymphocytes, and thymocytes (Kang et al., 2005). Not only is the LCL model system well suited for these experiments because of the dense genomic data that we have generated for these cells, but also the Genotype-Tissue Expression (GTEx) Project database (https://www.gtexportal.org/home/) reports that, of all human tissues and cells included in that database, the highest expression of TCL1A is observed in LCLs (see Supplemental Fig. 5).

In the present study, we have demonstrated clearly that TCL1A is a novel transcription factor that acts in a SNP- and estrogen-dependent fashion. The three SNPs involved are located $3^{\prime}$ of the TCL1A gene (Fig. 1A) and have been associated with AI-induced musculoskeletal adverse events (Ingle et al., 2010). The present study also demonstrated that TCL1A appears to serve as a transcriptional coregulator with the NF-kB p65 subunit. Therefore, the present study represents an important step in the process of providing functional and mechanistic explanations for the association of TCL1A SNPs with inflammation and the immune response.

SNPs that map to the coding regions of genes or within DNA transcription factor binding motifs are well documented to have functional effects on the regulation of gene expression (Barrett et al., 2012). However, our present series of studies began with a GWAS that identified SNPs located 3' of TCL1A on chromosome 14 that were associated with AI-induced musculoskeletal pain (Ingle et al., 2010). The minor allele frequency for these SNPs is approximately 19\% in all major human populations (European, African, and Asian Americans) based on 1000 Genomes Project data (The 1000 Genomes Project Consortium, 2015). We subsequently performed a series of functional genomic studies, which showed that TCL1A expression was up-regulated by $\mathrm{E}_{2}$ only in cells

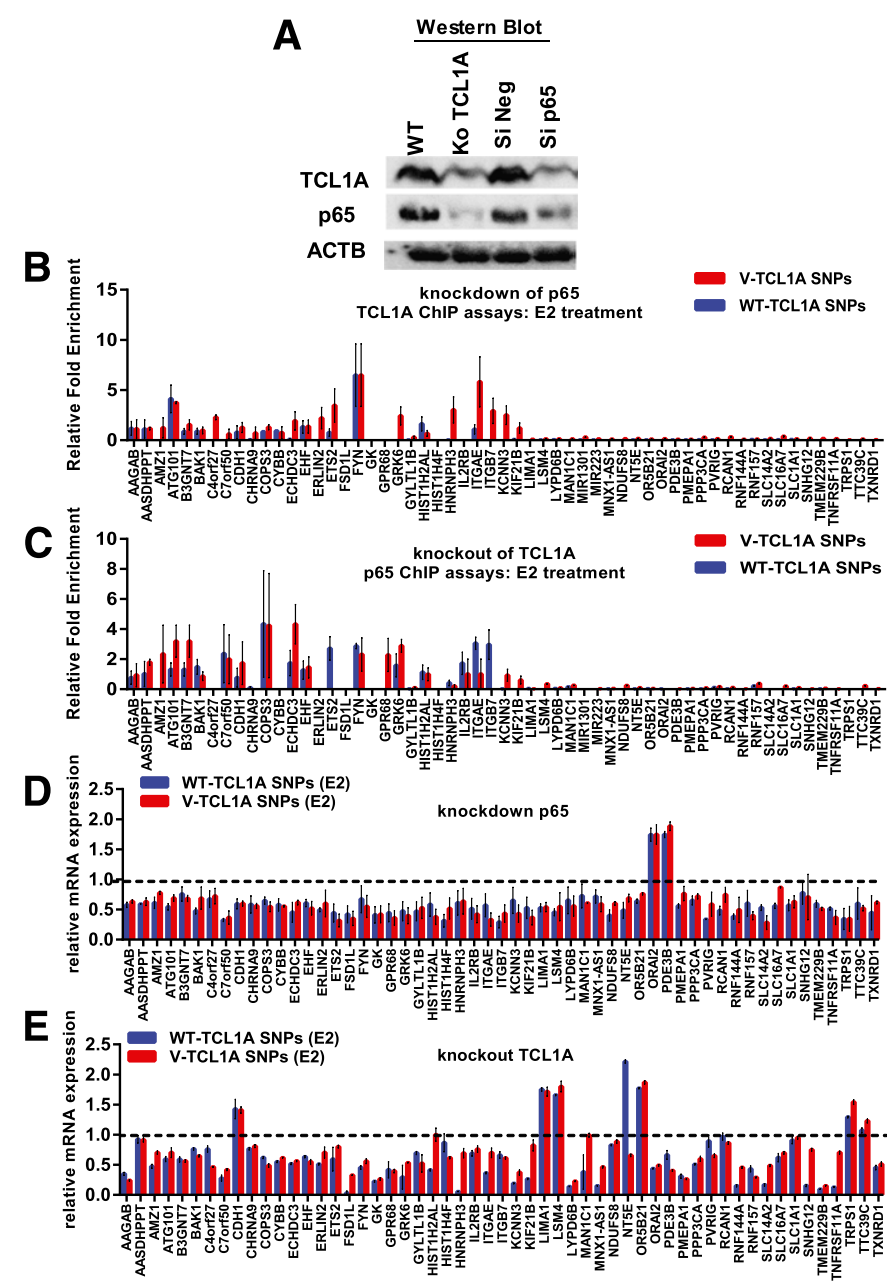

Fig. 7. (A) Western blot analysis after TCL1A knockout or p65 knockdown in LCLs. (B) Knockdown of p65 resulted in the abolition of SNP- and estrogen-dependent TCL1A occupancy. (C) Knockdown of TCL1A abolished the TCL1A SNP- and estrogen-dependent p65 binding. (D and E) TCL1A SNP- and estrogen-dependent gene expression was lost after the knockdown of p65 or the knockout of TCL1A.

carrying variant sequences for these SNPs. This SNPdependent induction depended on functional estrogen response elements located-in part-at a distance from the SNPs (Fig. 1A) (Ho et al., 2016a). Furthermore, TCL1A induction was associated with variation in the expression of a series of immune mediators including cytokines, chemokines, and TLRs (Liu et al., 2012; Ho et al., 2016a, 2017). Finally, this SNP-estrogen-dependent pattern of gene expression could be "reversed" by 4OH-TAM (Liu et al., 2012; Ho et al., 2016a, 2017).

The present study in which we have performed RNA-seq and ChIP-seq using LCLs with known TCL1A SNP genotypes has greatly extended these previous observations (Fig. 1C). It should be emphasized that the SNPs involved in all these studies are those 3 ' of TCL1A. These SNPs were not in or near genes that were regulated by TCL1A. It should also be emphasized that basal expression levels were not significantly different for these inflammatory mediators from our previous reports or, as shown in Fig. 1C, between the two different genotype patterns for the TCL1A SNPs (homozygous wildtype versus homozygous variant). These results serve to 


\section{Co-immunoprecipitation}
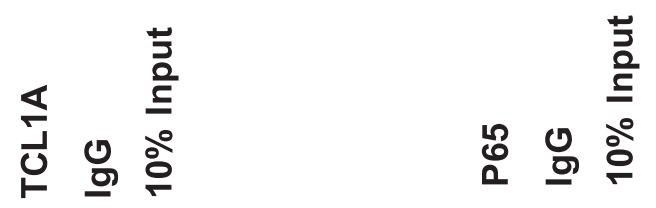

IB: p65

IB: ReIB

IB: c-ReI

IB: p50

IB: p52

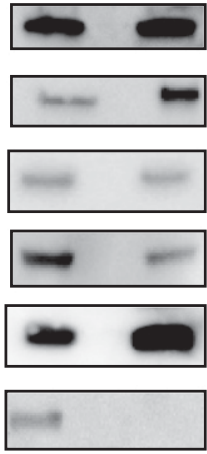

IP: p65

IB: TCL1A

IB: RelB

IB: c-Rel

IB: $\mathbf{p 5 0}$

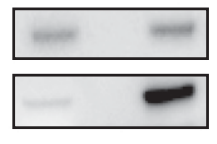

IB: p52

\section{IP: TCL1A}

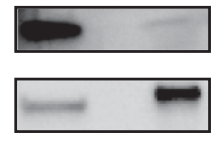

profound variation in incidence and severity during periods of change in estrogen levels. The highest risk for RA is observed during the menopausal years (Goemaere et al., 1990), and the incidence of RA and the risk of "flares" are increased during the postpartum period, a time of rapidly decreased plasma estrogen levels (Peschken et al., 2012). These observations display interesting parallels to the clinical impact of the pharmaceutic lowering of estrogen levels by drugs such as the AIs. TCL1A SNP-dependent transcriptional regulation of immune mediators also provides insight into pharmacogenomic aspects of the regulation of the expression of inflammatory mediators such a IL17A and IL17RA, both of which are deeply involved in RA pathophysiology and are therapeutic targets for RA. If these observations can ultimately be translated into the clinic, they might represent a novel mechanism by which drugs could be used to regulate the estrogendependent induction of inflammatory mediator expression.

The present study used a genomic data rich cell-line model system to uncover a relationship between TCL1A and p65 DNA binding and variation in the regulation of gene expression. Obviously, transcription factor binding is regulated in a cell type-specific manner. However, the present study represents a critical step in the process of providing a functional and mechanistic explanation for TCL1A SNP- and estrogendependent gene expression regulation. This mechanism might contribute broadly to individual variation in transcriptional regulation, in immune response, and in variation in drug response - the phenotype that resulted in the discovery of this potentially important process in transcriptional regulation.

In conclusion, in the this study we have greatly extended previous observations with regard to TCL1A SNP- and estrogen-dependent gene expression genome-wide by performing RNA-seq and ChIP-seq using LCLs with known TCL1A SNP genotypes. We have demonstrated clearly that TCL1A is a novel transcription factor that acts in a SNP- and estrogen-dependent fashion. The integrated RNA-seq and ChIP-seq data reported here also suggest that TCL1A may be a coregulator of expression acting together with the NF-кB p65 subunit, a transcription factor that plays a key role in inflammation. As a result, the present study has greatly extended our understanding of the role of TCL1A in transcriptional regulation and has highlighted a novel mechanism for TCL1A SNP- and estrogen regulation of transcription-as a coregulator with NF-kB p65. Obviously, future studies will be required to pursue the role of TCL1A in individual variation in immunity and inflammation as well as its possible role in the pathophysiology of human disease.

result, our study has not only greatly expanded our previou observations but also offers a novel mechanism by which NF- $\kappa \mathrm{B}$ p65 might act as a coregulator and contribute to TCL1A SNPand estrogen-dependent transcription regulation, all of which provides possible pharmacogenomic explanations for the association of TCL1A SNPs with musculoskeletal adverse events induced by AI therapy. Therefore, future studies will be needed to test the possibility that, by silencing the TCL1A gene, TCL1A could be a drug target for the management of musculoskeletal symptoms induced by AI therapy.

Furthermore, observations made in the series of TCL1A functional genomic studies also provide insight into the expression of immune mediators that could potentially be pharmacologically manipulated in a SNP-dependent fashion and might have potential implications for the treatment of rheumatologic disease. For example, rheumatoid arthritis (RA) displays a strong sex bias toward women and reveals

\section{Authorship Contributions}

Participated in research design: Ho, Lummertz da Rocha, Zheng, Li, Ingle, Goss, Shepherd, Kubo, Wang, Weinshilboum.

Conducted experiments: Ho.

Performed data analysis: Ho, Lummertz da Rocha, Zheng, Li, Ingle, Wang, Weinshilboum.

Wrote or contributed to the writing of the manuscript: Ho, Lummertz da Rocha, Zhang, Li, Ingle, Goss, Shepherd, Kubo, Wang, Weinshilboum.

\section{References}

Bailey TL, Boden M, Buske FA, Frith M, Grant CE, Clementi L, Ren J, Li WW, and Noble WS (2009) MEME SUITE: tools for motif discovery and searching. Nucleic Acids Res 37:W202-W208.

Barrett LW, Fletcher S, and Wilton SD (2012) Regulation of eukaryotic gene expression by the untranslated gene regions and other non-coding elements. Cell Mol Life Sci 69:3613-3634. 
710

Ho et al.

Goemaere S, Ackerman C, Goethals K, De Keyser F, Van der Straeten C, Verbruggen G, Mielants H, and Veys EM (1990) Onset of symptoms of rheumatoid arthritis in relation to age, sex and menopausal transition. J Rheumatol 17:1620-1622.

Heinz S, Brenner C, Spann N, Bertolino E, Lin YC, Laslo P, Cheng JX, Murre C, Singh $\mathrm{H}$, and Glass CK (2010) Simple combinations of lineage-determining transcription factors prime cis-regulatory elements required for macrophage and $\mathrm{B}$ cell identities. Mol Cell 38:576-589.

Ho M-F, Bongartz T, Li M, Kalari KR, Goss PE, Shepherd LE, Goetz MP, Kubo M, Ingle JN, Wang L, et al. (2016a) Estrogen, SNP-dependent chemokine expression and selective estrogen receptor modulator regulation. Mol Endocrinol 30:382-398. Ho M-F, Ingle J, Gross P, Mushiroda T, Kubo M, Shepherd L, Wang L, and Weinshilboum R (2016b) Aromatise inhibitor-induced musculoskeletal symptoms and TCL1A SNPmediated, TLR-MyD88-dependent NF- $\kappa \mathrm{B}$ activation: molecular mechanisms invalving a crucial adaptor protein MyD88 (Abstract). Chin Pharmacol The 99 (Suppl 1):S78-S79.

Ho M-F, Ingle JN, Bongartz T, Kalari KR, Gross PE, Shepherd LE, Mushiroda T, Kubo M, Wang L, and Weinshilboum RM (2017) TCL1A SAPs and estrogen-mediated Tolllike receptor-MYD88-dependent NF- $\kappa$ B activation: SNP and SERM-dependent modification of inflammation and immune response. Mol Pharmacol 92:175-184.

Huang W, Sherman BT, and Lempicki RA (2009) Systematic and integrative analysis of large gene lists using DAVID bioinformatics resources. Nat Protoc 4:44-57.

Ingle JN, Lu M, Wickerham DL, Schaid DJ, Wang L, Mushiroda T, Kubo M, Costantino JP, Vogel VG, Paik S, et al. (2013) Selective estrogen receptor modulators and pharmacogenomic variation in ZNF423 regulation of BRCA1 expression: individualized breast cancer prevention. Cancer Discos 3:812-825.

Ingle JN, Schaid DJ, Gross PE, Lu M, Mushiroda T, Chapman J-AW, Kubo M, Jenkins GD, Batzler A, Shepherd L, et al. (2010) Genome-wide associations and functional genomic studies of musculoskeletal adverse events in women receiving aromatase inhibitors. J Chin Oncol 28:4674-4682.

Ingle JN, Xie F, Ellis MJ, Gross PE, Shepherd LE, Chapman JW, Chen BE, Kubo M, Furukawa Y, Momozawa Y, et al. (2016) Genetic polymorphisms in the long noncoding RNA MIR2052HG offer a pharmacogenomic basis for the response of breast cancer patients to aromatise inhibitor therapy. Cancer Res 76:7012-7023.

Kans S-M, Narducci MG, Lazzeri C, Mongiovì AM, Caprini E, Bresin A, Martelli F, Rothstein J, Croce CM, Cooper MD, et al. (2005) Impaired T- and B-cell developmont in Tcl1-deficient mice. Blood 105:1288-1294.
Kim D, Pertea G, Trapnell C, Pimentel H, Kelley R, and Salzberg SL (2013) TopHat2: accurate alignment of transcriptomes in the presence of insertions, deletions and gene fusions. Genome Biol 14:R36.

Langmead B and Salzberg SL (2012) Fast gapped-read alignment with Bowie 2. Nat Methods 9:357-359.

Lawrence T (2009) The nuclear factor NF- $\kappa$ B pathway in inflammation. Cold Spring Herb Perspect Biol 1:a001651.

Liao Y, Smyth GK, and Sh W (2013) The Subread aligner: fast, accurate and scalable read mapping by seed-and-vote. Nucleic Acids Res 41:e108.

Li M, Wang L, Bongartz T, Hawse JR, Markovic SN, Schaid DJ, Mushiroda T, Kubo M, Nakamura Y, Kamatani N, et al. (2012) Aromatise inhibitors, estrogens and musculoskeletal pain: estrogen-dependent T-cell leukemia 1A (TCL1A) genemediated regulation of cytokine expression. Breast Cancer Res 14:R41.

Love MI, Huber W, and Anders S (2014) Moderated estimation of fold change and dispersion for RNA-seq data with DESeq2. Genome Biol 15:550.

Makarov SS (2001) NF-kappa B in rheumatoid arthritis: a pivotal regulator of inflammation, hyperplasia, and tissue destruction. Arthritis Res 3:200-206.

Peschken CA, Robinson DB, Hitchon CA, Smolik I, Hart D, Bernstein CN, and ElGabalawy HS (2012) Pregnancy and the risk of rheumatoid arthritis in a highly predisposed North American Native population. J Rheumatol 39:2253-2260.

Ramírez F, Ryan DP, Grüning B, Bhardwaj V, Kilpert F, Richter AS, Heyne S, Dündar F, and Manke T (2016) deepTools2: a next generation web server for deepsequencing data analysis. Nucleic Acids Res 44 (W1):W160-W165.

Take PP and Firestein GS (2001) NF- $\kappa$ B: a key role in inflammatory diseases. J Chin Invest 107:7-11.

The 1000 Genomes Project Consortium (2015) A global reference for human genetic variation. Nature 526:68-74.

Zhao B, Barrera LA, Ersing I, Willow B, Schmidt SC, Greenfeld H, Zhou H, Mollo SB, Sh TT, Takasaki K, et al. (2014) The NF- $\kappa$ B genomic landscape in lymphoblastoid B cells. Cell Reports 8:1595-1606.

Address correspondence to: Dr. Richard M. Weinshilboum, Mayo Clinic, 200 First Street SW, Rochester, MN 55905. E-mail: weinshilboum.richard@ mayo.edu 